\title{
Presepsin as a Biomarker for Risk Stratification and Prognosis of Acute Cholangitis:A Single Center Prospective Cohort Study
}

\section{Hanyu Zhang}

Capital Medical University Affiliated Beijing Friendship Hospital https://orcid.org/0000-0002-1865047X

\section{Zhaoqing Lu}

Capital Medical University Affiliated Beijing Friendship Hospital

\section{Guoxing Wang}

Capital Medical University Affiliated Beijing Friendship Hospital

\section{Di Wu}

Capital Medical University Affiliated Beijing Friendship Hospital

\section{Xu Ge}

Capital Medical University Affiliated Beijing Friendship Hospital

\section{Miaorong Xie}

Capital Medical University Affiliated Beijing Friendship Hospital

Chunsheng Li ( $\sim$ lcscyyy@163.com)

Capital Medical University Affiliated Beijing Friendship Hospital https://orcid.org/0000-0002-31614457

\section{Research}

Keywords: C-creative protein (CRP), procalcitonin (PCT), presepsin, acute cholangitis, mortality

Posted Date: April 19th, 2021

DOl: https://doi.org/10.21203/rs.3.rs-412125/v1

License: (c) (i) This work is licensed under a Creative Commons Attribution 4.0 International License. Read Full License 


\section{Abstract}

Background: Presepsin is currently a promising biomarker for the early diagnosis and prognosis of acute bacterial infection. Acute cholangitis is caused by bacterial infection and has high morbidity and mortality. The study engaged to assess the grading and prognostic value of presepsin in patients with acute cholangitis.

Methods: This study enrolled patients with acute cholangitis in the emergency department from May 1 , 2019 to December 20, 2020. The patients were evaluated for severity by the 2018 Tokyo Guidelines for Acute Cholangitis. Patients' baseline features and routine clinical data were collected. On admission, presepsin, procalcitonin (PCT) and systemic inflammatory response syndrome (SIRS) and sequential organ failure assessment (SOFA) scores were determined; and blood cultures were performed. IBM SPSS software (version 22.0) was used. The comparation of values was performed by Pearson chi-square test or Fisher's exact test or Mann-Whitney $U$ test. The area under the receiver operating characteristic curve (AUC), multivariate logistic regression, and correlation analysis were used to analyze this importance of determining the presepsin levels on admission for acute cholangitis.

Results: In total, 330 patients, including 109, 101, and 120 patients classified as having mild, moderate, and severe cholangitis, respectively, were examined. The AUCs of presepsin were 0.713 in predicting severe acute cholangitis, better than those of white blood cell (WBC 0.411), C-reactive protein (CRP $0.615)$, PCT 0.608 , and total bilirubin (T-Bil 0.441) $(P<0.05)$. The AUC of presepsin in predicting 28-day mortality was higher than that of WBC, CRP, PCT, and T-Bil. The presepsin level in the positive blood culture group was higher than that in the negative blood culture group $(P=0.000)$. The $P$ values for correlations of presepsin with SIRS and SOFA scores were 0.002 and 0.000 , respectively.

Conclusions: Presepsin levels on admission were correlated with SIRS and SOFA scores. Presepsin may predict positive blood culture and 28-day mortality in patients with acute cholangitis, and it is superior to WBC, CRP, PCT, and T-Bil in the risk stratification and prognostic assessment of severe cholangitis.

Trial registration: Ethical code of this study is 2018-P2-063-01 and acquired on May, 22, 2018.

\section{Introduction}

Acute cholangitis is an acute inflammatory reaction caused by a bacterial infection of the biliary tract [1]. It rapidly progresses and can develop into sepsis or septic shock [2]. Acute cholangitis is currently diagnosed based on clinical symptoms and signs, white blood cell (WBC) counts, C-reactive protein (CRP) and total bilirubin (T-Bil) levels, and imaging findings. The mortality rate of patients with acute severe cholangitis ranges from $2.7-10 \%[1,3]$. Thus, early risk stratification and prognostic assessments are important in the emergency treatment of acute cholangitis; however, an ideal predictive biomarker remains unknown. 
The effectiveness of presepsin as an infectious biomarker to grade the early severity of acute cholangitis remains unclear [4]. Therefore, this prospective cohort study aimed to compare levels of presepsin with traditional biomarkers in patients with acute cholangitis on admission to evaluate the role of presepsin in the early risk stratification and prognostic assessment of acute cholangitis.

\section{Methods}

\section{Study design}

This clinical study was carried out in Beijing Friendship Hospital, a 2000-bed teaching hospital with approximately 200,000 emergency admissions per year. The Medical Ethics Committee of Beijing Friendship Hospital approved this study (Ethical code: 2018-P2-063-01). All patients or their relatives signed the informed consent form prior to study participation.

\section{Inclusion And Exclusion Criteria>}

All patients included in this study met the diagnostic criteria for acute cholangitis according to the 2018 Tokyo Guidelines for Acute Cholangitis (TG18) [5]. The diagnostic criteria were as follows: (1) systemic inflammation (fever/chill or abnormal WBC counts or elevated CRP levels); (2) cholestasis (T-Bil levels $\geq$ $34.2 \mu \mathrm{g} / \mathrm{mL}$ or liver enzyme levels $>1.5$ times of the upper normal limit); (3) supporting imaging findings (imaging evidence or etiology of biliary dilatation, such as benign or malignant stenosis, stone, and stent). Participants were excluded if they had liver diseases, such as viral or drug hepatitis, chronic liver disease, chronic renal insufficiency, or any other infections on admission.

\section{Patients}

Overall, this study enrolled 330 patients (age $>18$ years) diagnosed with acute cholangitis from May 1 , 2019 to December 20, 2020. All patients were administered intravenous antibiotics (imipenem and cilastatin in sodium for injection $0.5 \mathrm{~g}$ every 8 hours; or cefoperazone sodium and sulbactam sodium 3.0 g every 12 hours; or levofloxacin $0.5 \mathrm{~g}$ once daily; or tazobactam sodium/piperacillin sodium for injection $4.5 \mathrm{~g}$ every 8 hours) as soon as acute cholangitis was diagnosed according to the TG18.

\section{Examinations}

On admission, whole blood ( $30 \mathrm{~mL}$ ) was collected from the patients' cubital vein, and the systemic inflammatory response syndrome (SIRS) [6] and sequential organ failure assessment (SOFA) scores [7] were also determined immediately. Blood was tested for the following parameters: WBC, CRP, platelet, procalcitonin (PCT), presepsin, T-Bil, total protein (TP), albumin (A), alanine aminotransferase, aspartate aminotransferase, lactate dehydrogenase, myocardial enzyme, serum creatinine, and international 
normalized ratio. Blood culture was also performed. Furthermore, $1.5 \mathrm{~mL}$ of arterial blood was collected for blood gas analysis.

\section{Data Collection}

Clinical data, including baseline demographic features and information related to underlying disease, composite complication index (CCl), antibiotic or fluid resuscitation before enrollment, laboratory parameters, and imaging, were collected on admission. Data regarding vital signs, mental status, oxygen flow, hepatic function, biomarkers, blood culture, urinary output, vasoactive drugs (dopamine, dobutamine, adrenaline, and norepinephrine), arterial partial pressure of oxygen, and SIRS and SOFA scores were also collected on admission.

\section{Measurement}

Presepsin levels were measured using the PATHFAST analyzer (PATHFAST ${ }^{\mathrm{TM}}$ presepsin, Mitsubishi Chemical Medience Corporation, Tokyo, Japan), which is a chemiluminescence immunoassay of venous whole blood based on ethylene diamine tetraacetic acid as an anticoagulant [8]. The detection range of presepsin level was $20-200,000 \mathrm{pg} / \mathrm{mL}$. PCT levels were assayed with a BioMerieux Mini VIDAS immunoassay analyzer (Block Scientific, Bohemia, NY) from the plasma obtained from blood centrifuged for $15 \mathrm{~min}$ at $1000 \times \mathrm{g}$. The detection range of PCT level was $0.05-200 \mathrm{ng} / \mathrm{mL}$. Information regarding other indicators were obtained from the clinical laboratory data.

\section{Severity Of Acute Cholangitis Based On The Tg18}

Severe acute cholangitis was diagnosed if any dysfunction was noted in the cardiovascular, nervous, respiratory, or blood systems, kidneys, or liver. Moderate acute cholangitis was defined if any two of the following conditions were observed: deviant WBC counts, high fever, age $>75$ years, hyperbilirubinemia, and hypoalbuminemia. Mild acute cholangitis was diagnosed if the criteria for the above two grades of acute cholangitis was not met.

\section{Statistical Analyses}

IBM SPSS version 22.0 software was used for whole statistical analyses. Two-sided $P$ value $<0.05$ was considered statistically significant, and the significant level for pairwise comparisons among three groups was set to $0.017(P=0.05 / 3)$. Classified variables were expressed in counts and percentages and were compared using the Pearson chi-square test or Fisher's exact test. Skewed distribution variables were expressed in medians (25-75th percentiles), and Mann-Whitney $U$ test was used for comparison between groups. Diagnostic sensitivity, specificity, accuracy, critical value, and area under the receiver operating characteristic (ROC) curve (AUC) were determined by performing a ROC curve analyses to verify 
the role of determining presepsin levels on admission for acute cholangitis. The optimal critical value was calculated using the Youden index according to the ROC curves. The correlation among the main research indicators was determined using Pearson correlation. Multivariate logistic regression analysis was applied for determining the risk factors for 28-day mortality.

\section{Results}

Among the 350 patients with acute cholangitis who were screened, 20 didn't agree to take part in this study; finally, 330 or their relatives signed the informed consent form prior to this study. All patients with acute cholangitis on admission were divided into mild, moderate, and severe groups based on the TG18. The numbers of patients in the above three groups were 109 (33.03\%), 101 (30.61\%), and 120 (36.36\%), respectively (Fig. 1).

The features and outcomes of 330 patients with acute cholangitis are shown in Table 1, including 197 men and 133 women. The median age was 74 years. Age, WBC, CRP, PCT, presepsin, T-Bil, D-Bil, CCl, and SIRS and SOFA scores of moderate and severe cholangitis patients were higher than those of mild cholangitis patients, whereas the opposite was true for TP and A. Clinically, patients with severe cholangitis were more likely to be administered carbapenems. Cephalosporins and quinolones were only given for patients with mild acute cholangitis. Eight deaths occurred within 28 days in patients with severe cholangitis. The 28 -day mortality rate of patients with acute cholangitis was $2.42 \%$.

\section{Role of WBC, CRP, PCT, presepsin, and T-Bil in the severity grading of acute cholangitis}

WBC, CRP, PCT, presepsin, T-Bil, and SIRS and SOFA scores of patients with moderate and severe cholangitis were higher than those of patients with mild cholangitis (all $P=0.000$; Table 1). Presepsin had the best $P$ value in patients with mild-to-moderate and moderate-to-severe acute cholangitis (Fig. 2a). The AUC of presepsin in predicting moderate acute cholangitis was 0.726 , which was prominently better than that of $\operatorname{CRP}(0.611, P=0.014)$ and PCT $(0.610, P=0.006)$. No differences in the AUC of T-Bil $(0.680, P=$ $0.287)$ and WBC $(0.756, P=0.51)$ were observed. The optimal critical value of presepsin for grading moderate acute cholangitis was $1970 \mathrm{pg} / \mathrm{mL}$ (sensitivity, 0.73; specificity, 0.66; and accuracy, 0.70) ((Fig. 3a). The AUC of presepsin in predicting severe acute cholangitis was 0.713 , which was higher than that of WBC (0.411, $P=0.002), \operatorname{CRP}(0.615, P=0.021)$, PCT $(0.608, P=0.009)$, and T-Bil $(0.441, P=0.008)$. The optimal critical value of presepsin for grading severe acute cholangitis was $3099 \mathrm{pg} / \mathrm{mL}$ (sensitivity, 0.90; specificity, 0.51; and accuracy, 0.69) (Fig. 3b).

\section{Presepsin as a biomarker for28-day mortality in patients with acute cholangitis}

Eight patients died within 28 days. Presepsin levels in the survival group were lower than those in the death group $(P=0.015)$, although no significant difference was observed in the WBC count, PCT and T-Bil levels in both groups $(P>0.05)$. As expected, multivariate logistic regression demonstrated an increasing likelihood of 28-day mortality with presepsin (odds ratio [OR], 1; 95\% confidence interval [Cl], 1.00-1.01; $P$ $=0.001$ ). Although there was no significant difference in $P$ values, the AUC of presepsin in predicting 28- 
day mortality was 0.752 , which was higher than that of WBC (0.602), CRP (0.704), PCT (0.637), and T-Bil (0.552). The optimal critical value of presepsin to predict 28-day mortality was $3333 \mathrm{pg} / \mathrm{mL}$ (Fig. 2b, Fig. 3c).

\section{Presepsin as a biomarker of positive blood culture in patients with acute cholangitis}

WBC, PCT, and presepsin levels were greater in the positive blood culture group than in the negative blood culture group $(P<0.001)$; however, CRP and T-Bil levels did not show significant difference in both groups $(P>0.05)$. The ROC curves of biomarkers showed that PCT had the best AUC (0.705), but there was no difference observed between the AUC of PCT and presepsin in predicting positive blood culture $(P=$ 0.127). This could indicate a positive blood culture when the presepsin level was $>2222 \mathrm{pg} / \mathrm{mL}$ (Fig. 2c, Fig. 3d).

\section{Presepsin was related to T-Bil, WBC, CRP, PCT, and SOFA and SIRS scores}

Multivariate analysis revealed that presepsin was closely related to SOFA score $(P<0.01)$. The $P$ value for the correlation between presepsin and SOFA score was $0.000\left(r=0.498,95 \% \mathrm{Cl} 0.4116-0.5755, R^{2}=\right.$ 0.248) (Fig. 4). The correlation between WBC, CRP, PCT, presepsin and T-Bil revealed that only presepsin was correlated with T-Bil $(P=0.000)$ (Fig. 5). Presepsin was correlated with WBC, CRP, PCT, and SIRS scores (Table 2).

\section{Discussion}

For a long time, acute cholangitis has been diagnosed based on the Charcot's triad; however, its misdiagnosis rate was found to be 30-80\% [9-11]. With the introduction of WBC, CRP, and T-Bil into the TG07, TG13, and TG18, the diagnosis rate improved, but some cases are still misdiagnosed and early risk stratification assessments are difficult [12]. PCT has been recommended as a biomarker for predicting severe acute cholangitis; however, PCT levels are increased in patients with trauma [13] and adrenocortical failure [14] and are falsely negative in patients with atypical bacterial infections [15]. Early diagnosis and grading of acute cholangitis are still important clinical problems. Presepsin is a promising biomarker of bacterial infectious diseases discovered in recent years, and its sensitivity and accuracy are better than those of PCT, WBC, and CRP [16-18]. Based on the abovementioned findings, we demonstrated that presepsin had higher sensitivity and specificity in risk stratification and prognosis of acute cholangitis and had the same positive rate in predicting bacterial culture as PCT.

SOFA score $\geq 2$ is the diagnostic criterion for sepsis in the new sepsis-3.0 definitions, which shows the number and severity of organ dysfunction [19]. In this study, presepsin levels in patients with acute cholangitis were closely related to SIRS and SOFA scores $(P<0.002)$. On comparing presepsin with PCT, WBC, CRP, and T-Bil, we found that presepsin had the best predictive value for determining the severity of cholangitis (all $P<0.01$ ). This suggests that presepsin levels could determine the severity of acute cholangitis to some extent. 
Most cases with acute cholangitis are associated with intestinal bacterial infection and elevated bile acid and bilirubin levels, which play a key role in innate immune function activation [20] and improper cytokine or antigen secretion in mononuclear/kupffer cells [21]. CRP and PCT levels are indirect acute-phase biomarkers of host-pathogen systemic responses, because their syntheses require interleukin-1 and interleukin-6, which are secreted by monocytes/macrophages; however, their synthesis is suppressed in patients with acute cholangitis [21]. Presepsin is an infection-specific biomarker for innate immune responses that can directly cleave from the monocyte/macrophage-specific CD14 receptor complex once germs or lipopolysaccharide invade the bloodstream of an organism [22, 23]. In patients with acute cholangitis, presepsin may be excessively secreted in the peripheral blood because of monocyte endocytosis, which reduces the abundance of CD14 on the cell membrane [24].

In this study, the overall 28-day mortality rate was $2.42 \%$, which was lower than the reported mortality rate of $2.7-10 \%$ for acute severe cholangitis $[1,3]$ and may be related to the aggressive and comprehensive treatment and shorter observation time. Some studies showed that presepsin levels have significant prognostic values for mortality risk in patients with sepsis, sepsis-related diseases, or bacterial infections $[16,25,26]$. Some studies also used WBC counts and T-Bil levels as predictors of mortality in patients with acute cholangitis [27]. In this study, presepsin was more positively correlated with SOFA scores, WBC counts, and T-Bil levels. Multivariate logistic regression demonstrated an increasing likelihood of 28-day mortality with increasing presepsin levels in patients with acute cholangitis, whereas WBC counts and CRP and PCT levels were not associated with 28-day mortality. On comparing the AUCs of presepsin with those of WBC, CRP, PCT, and T-Bil in predicting 28-day mortality, although there was no statistical difference, presepsin was found to have the best AUC. Therefore, presepsin levels on admission could help clinicians determine the early risk of death in patients with cholangitis.

Blood cultures are the gold standard for diagnosing

bloodstream infectious diseases; however, blood cultures are often negative, and the results are usually obtained after several days [28]. PCT is a biomarker associated with positive blood cultures [29, 30]. As a biomarker of infection, presepsin was superior to PCT in risk stratification and prognostic assessment of acute cholangitis and was as good as PCT in predicting positive bacterial cultures. Presepsin levels can be obtained several days earlier than blood culture, which facilitates early treatment to some extent.

Some limitations should be recognized in this study. Firstly, the study was performed at a single hospital with a relatively small sample size; furthermore, there could be some bias in terms of the enrolled patients, and thus, multicenter and large sample studies need to be conducted in the future. Secondly, the etiology and pathogens that were not statistically analyzed could interfere with the presepsin levels in our patients. Thirdly, the duration of illness and previous treatment effects were not considered when patients were enrolled, which could have affected the observation of biomarkers.

\section{Conclusions}


Presepsin levels predict the severity of acute cholangitis, positive blood culture, and 28-day mortality and are also correlated with SIRS and SOFA scores in patients with acute cholangitis. Early and timely determination of presepsin levels is helpful in risk stratification and prognostic assessment of acute cholangitis. Although the result was exciting, larger study should be performed to verify the role of presepsin in acute cholangitis.

\section{Abbreviations}

WBC: white blood cell; CRP: C-reactive protein; PCT, procalcitonin; AUC: area under the curve; IQR: interquartile range; ROC: receiver-operating characteristic; TG18: 2018 Tokyo guidelines; T-Bil: total bilirubin; TP: total protein; A: albumin; SIRS: systemic inflammatory response syndrome; SOFA: sequential organ failure assessment; $\mathrm{CCl}$ : composite complication index.

\section{Declarations}

\section{Acknowledgments}

We deeply appreciate the emergency staff, gastroenterologists, hepatobiliary surgeons, radiologists, sonographers, and interventional physicians for their sincere.

\section{Disclaimer}

The opinions and assertions expressed herein are those of the author(s) and do not necessarily reflect the official policy or position of Beijing Friendship Hospital, Capital Medical University, China.

\section{Authors' contributions}

Hanyu Zhang, Miaorong Xie and Chunsheng Li designed the study. Hanyu Zhang, Zhaoqing Lu, Di Wu and Xu Ge collected sample and clinical data. Hanyu Zhang measured presepsin and analyzed all data and the statistical analysis. Guoxing Wang conducted clinical supervision. Final manuscript got common consents from all authors.

\section{Funding}

This study was supported by the National Natural Science Foundation of China (81773931 and 81374004), as well as the Beijing Municipal Administration of Hospitals Clinical Medicine Development of Special Funding Support "Yanfan" Project (ZYLX201802).

\section{Availability of data and materials}

The datasets analyzed during the current study are available from the corresponding author on reasonable request. 
In this study, ethical approval and sample consent were obtained by Guoxing Wang, et al. The Biobank of Beijing Friendship Hospital approved sample collection and the Medical Ethics Committee of Beijing Friendship Hospital approved this study. All participates gave informed consent.

\section{Consent for publication}

Not applicable.

\section{Competing interests}

The authors declare that they have no competing interests.

\section{Author details}

Department of Emergency Medicine, Beijing Friendship Hospital, Capital Medical University. 95 Yong-an Road, Xi-Cheng District, Beijing 100050, China.

\section{References}

1. Kimura Y, Takada T, Kawarada Y, Nimura Y, Hirata K, Sekimoto M, Yoshida M, Mayumi T, Wada K, Miura F, et al. Definitions, pathophysiology, and epidemiology of acute cholangitis and cholecystitis: Tokyo Guidelines. J Hepatobiliary Pancreat Surg. 2007;14(1):15-26.

2. Kimmings AN, van Deventer SJ, Rauws EAJ, Huibregtse K, Gouma DJ. Systemic inflammatory response in acute cholangitis and after subsequent treatment. Eur J Surg. 2000;166(9):700-5.

3. Welch JP, Donaldson GA. The urgency of diagnosis and surgical treatment of acute suppurative cholangitis. Am J Surg. 1976;131(5):527-32.

4. Lin J, Sun H, Li J, Zheng Y, Shao C, Zhang YH, Chang H. Role of Presepsin for the Assessment of Acute Cholangitis Severity. Clin Lab. 2016;62(4):679-87.

5. Yokoe M, Hata J, Takada T, Strasberg SM, Asbun HJ, Wakabayashi G, Kozaka K, Endo I, Deziel DJ, Miura F, et al. Tokyo Guidelines 2018: diagnostic criteria and severity grading of acute cholecystitis (with videos). J Hepatobiliary Pancreat Sci. 2018;25(1):41-54.

6. Brunkhorst FM, Wegscheider K, Forycki ZF, Brunkhorst R. Procalcitonin for early diagnosis and differentiation of SIRS, sepsis, severe sepsis, and septic shock. Intensive Care Med. 2000;26(Suppl 2):148-52.

7. Tavare A, O'Flynn N. Recognition, diagnosis, and early management of sepsis: NICE guideline. $\mathrm{Br} \mathrm{J}$ Gen Pract. 2017;67(657):185-6.

8. Novelli S, Morabito V, Ruberto F, Bini F, Marinozzi F, Pugliese F, Berloco P, Pretagostini R. Diagnostic Value of Presepsin for Bacterial Infection in Cirrhosis: A Pilot Study. Transplant Proc. 2020;52(5):1593-600.

9. Kiriyama S, Takada T, Strasberg SM, Solomkin JS, Mayumi T, Pitt HA, Gouma DJ, Garden OJ, Buchler MW, Yokoe M, et al. New diagnostic criteria and severity assessment of acute cholangitis in revised 
Tokyo Guidelines. J Hepatobiliary Pancreat Sci. 2012;19(5):548-56.

10. Kiriyama S, Takada T, Hwang TL, Akazawa K, Miura F, Gomi H, Mori R, Endo I, Itoi T, Yokoe M, et al. Clinical application and verification of the TG13 diagnostic and severity grading criteria for acute cholangitis: an international multicenter observational study. J Hepatobiliary Pancreat Sci. 2017;24(6):329-37.

11. O'Connor MJ, Schwartz ML, McQuarrie DG, Sumer HW. Acute bacterial cholangitis: an analysis of clinical manifestation. Arch Surg. 1982;117(4):437-41.

12. Gravito-Soares E, Gravito-Soares M, Gomes D, Almeida N, Tome L. Clinical applicability of Tokyo guidelines 2018/2013 in diagnosis and severity evaluation of acute cholangitis and determination of a new severity model. Scand J Gastroenterol. 2018;53(3):329-34.

13. Benoist JF, Mimoz O, Assicot M. [Procalcitonin in severe trauma]. Ann Biol Clin (Paris). 1998;56(5):571-4.

14. Hatherill M, Jones G, Lim E, Tibby SM, Murdoch IA. Procalcitonin aids diagnosis of adrenocortical failure. Lancet. 1997;350(9093):1749-50.

15. Baumgartner A, Schuetz P. [Laboratory results in clinical practice: importance of interpretation in the clinical context]. Ther Umsch. 2015;72(2):119-23.

16. Zhao J, Tan Y, Wang L, Shi Y. Discriminatory ability and prognostic evaluation of presepsin for sepsis-related acute respiratory distress syndrome. Sci Rep. 2020;10(1):9114.

17. Venugopalan DP, Pillai G, Krishnan S. Diagnostic Value and Prognostic Use of Presepsin Versus Procalcitonin in Sepsis. Cureus. 2019;11(7):e5151.

18. Unuma K, Makino Y, Sasaki Y, Iwase H, Uemura K. Presepsin: A potential superior diagnostic biomarker for the postmortem differentiation of sepsis based on the Sepsis-3 criteria. Forensic Sci Int. 2019;299:17-20.

19. Aliu-Bejta A, Atelj A, Kurshumliu M, Dreshaj S, Barsic B. Presepsin values as markers of severity of sepsis. Int J Infect Dis. 2020;95:1-7.

20. Liu R, Li X, Huang Z, Zhao D, Ganesh BS, Lai G, Pandak WM, Hylemon PB, Bajaj JS, Sanyal AJ, et al. C/EBP homologous protein-induced loss of intestinal epithelial stemness contributes to bile duct ligation-induced cholestatic liver injury in mice. Hepatology. 2018;67(4):1441-57.

21. Scott-Conner CE, Grogan JB. The pathophysiology of biliary obstruction and its effect on phagocytic and immune function. J Surg Res. 1994;57(2):316-36.

22. Arai Y, Mizugishi K, Nonomura K, Naitoh K, Takaori-Kondo A, Yamashita K. Phagocytosis by human monocytes is required for the secretion of presepsin. J Infect Chemother. 2015;21(8):564-9.

23. Chenevier-Gobeaux C, Borderie D, Weiss N, Mallet-Coste T, Claessens YE. Presepsin (sCD14-ST), an innate immune response marker in sepsis. Clin Chim Acta. 2015;450:97-103.

24. Li J, Tang Z, Xie M, Hang C, Yu Y, Li C. Association between elevation of plasma biomarkers and monocyte dysfunction and their combination in predicting sepsis: An observational single-centre cohort study. Innate Immun. 2020;26(6):514-27. 
25. Dragoescu AN, Padureanu V, Stanculescu AD, Chiutu LC, Florescu DN, Gheonea IA, Padureanu R, Stepan A, Streba CT, Drocas Al, et al: Presepsin as a Potential Prognostic Marker for Sepsis According to Actual Practice Guidelines. J Pers Med 2020, 11(1).

26. Ferrarese A, Frigo AC, Mion MM, Plebani M, Russo FP, Germani G, Gambato M, Cillo U, Cattelan A, Burra $\mathrm{P}$, et al: Diagnostic and prognostic role of presepsin in patients with cirrhosis and bacterial infection. Clin Chem Lab Med 2020.

27. Schwed AC, Boggs MM, Pham XD, Watanabe DM, Bermudez MC, Kaji AH, Kim DY, Plurad DS, Saltzman DJ, de Virgilio C. Association of Admission Laboratory Values and the Timing of Endoscopic Retrograde Cholangiopancreatography With Clinical Outcomes in Acute Cholangitis. JAMA Surg. 2016;151(11):1039-45.

28. Zadroga R, Williams DN, Gottschall R, Hanson K, Nordberg V, Deike M, Kuskowski M, Carlson L, Nicolau DP, Sutherland C, et al. Comparison of 2 blood culture media shows significant differences in bacterial recovery for patients on antimicrobial therapy. Clin Infect Dis. 2013;56(6):790-7.

29. Webb AL, Kramer N, Stead TG, Mangal R, Lebowitz D, Dub L, Rosario J, Tak M, Reddy S, Lee JR, et al. Serum Procalcitonin Level Is Associated with Positive Blood Cultures, In-hospital Mortality, and Septic Shock in Emergency Department Sepsis Patients. Cureus. 2020;12(4):e7812.

30. Yu Y, Li XX, Jiang LX, Du M, Liu ZG, Cen ZR, Wang H, Guo ZH, Chang P. Procalcitonin levels in patients with positive blood culture, positive body fluid culture, sepsis, and severe sepsis: a crosssectional study. Infect Dis (Lond). 2016;48(1):63-9.

\section{Tables}

Due to technical limitations, table 1 and 2 is only available as a download in the Supplemental Files section.

\section{Figures}




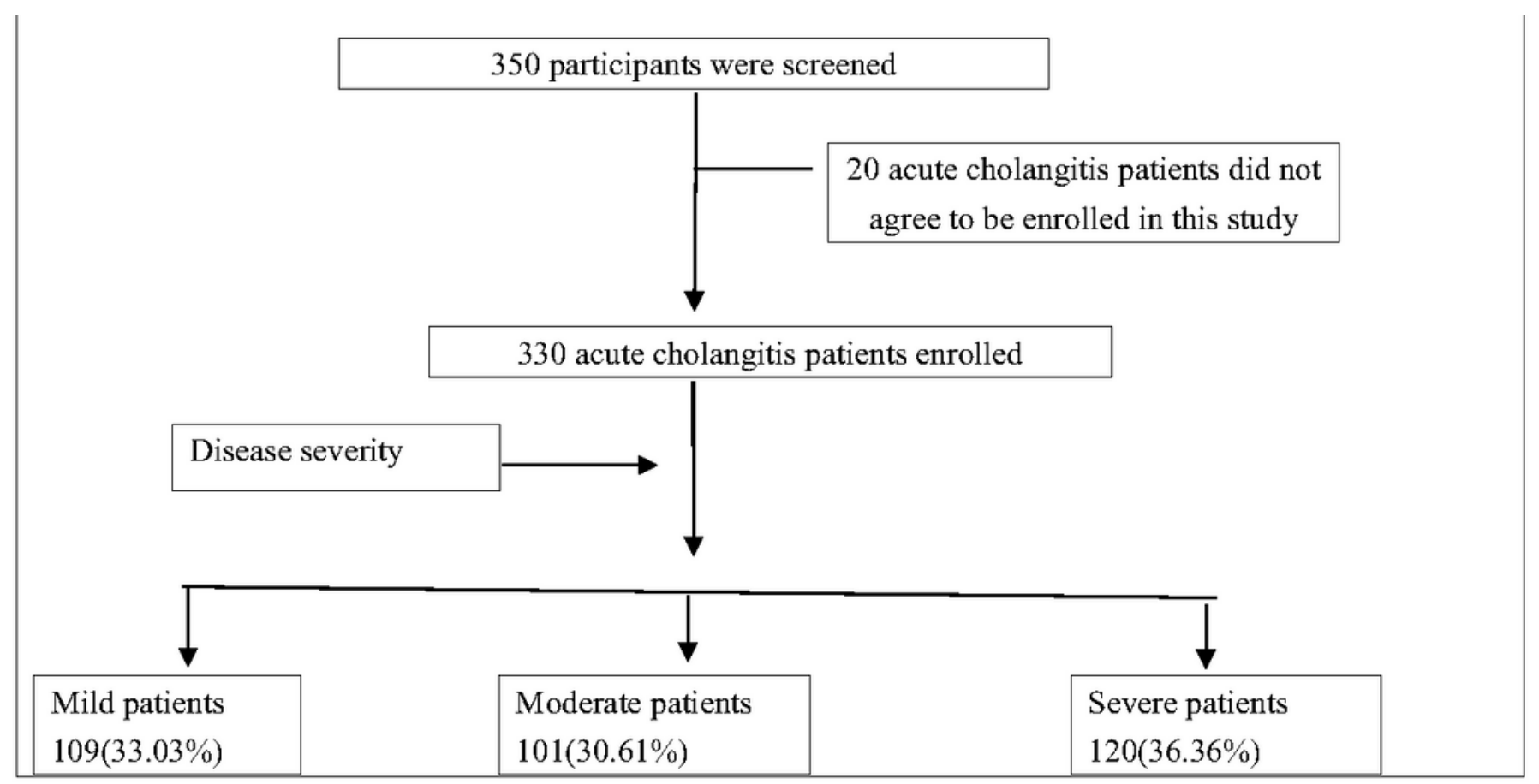

\section{Figure 1}

Flowchart for participants. Diagnostic criteria and severity grading of acute cholangitis was determined by the Tokyo guidelines 2018: diagnosis criteria and severity grading of acute cholangitis (TG18) 


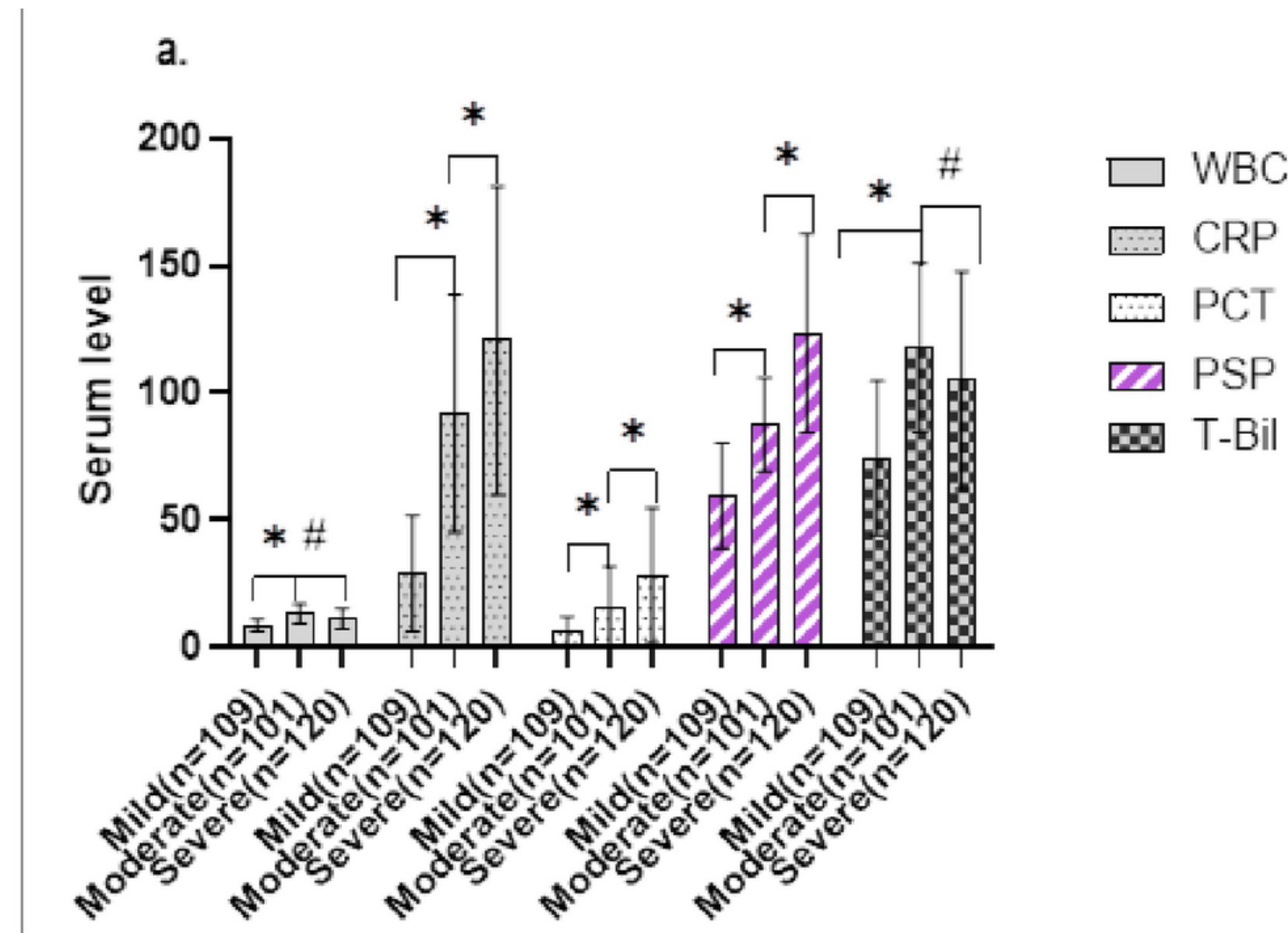

\section{Biomarkers in grading of acute cholangitis}

b.

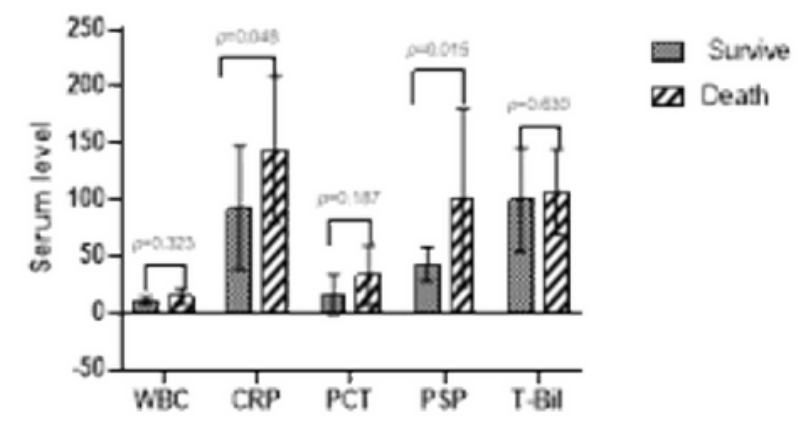

Biomarkers in survive group and death group of acute cholangitis c.

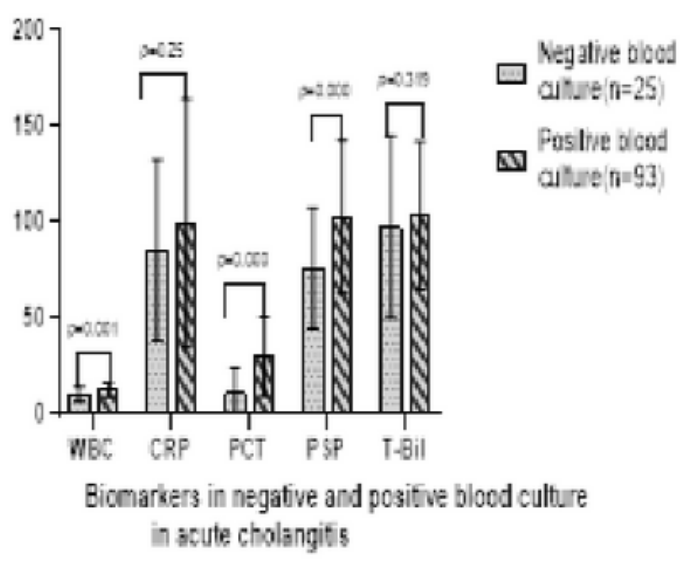

Figure 2

Biomarkeres in acute cholangitis. WBC white blood cell (x109/L), CRP C-reactive protein (mg/L), PCT procalcitonin $(\mathrm{ng} / \mathrm{mL})$, PSP presepsin $(\mathrm{pg} / \mathrm{mL})$, T-Bil total bilirubin $(\mu \mathrm{mol} / \mathrm{L}) .{ }^{*} p<0.01 ; \# p>0.017 . p<0.017$ was set to significant level in $a ; P<0.05$ was set to significant level in $b$ and $c$. The PSP value in the figure was the result of presepsin concentration divided by 3 . 
a. ROC curve: ROC of mild and moderate acute cholangitis

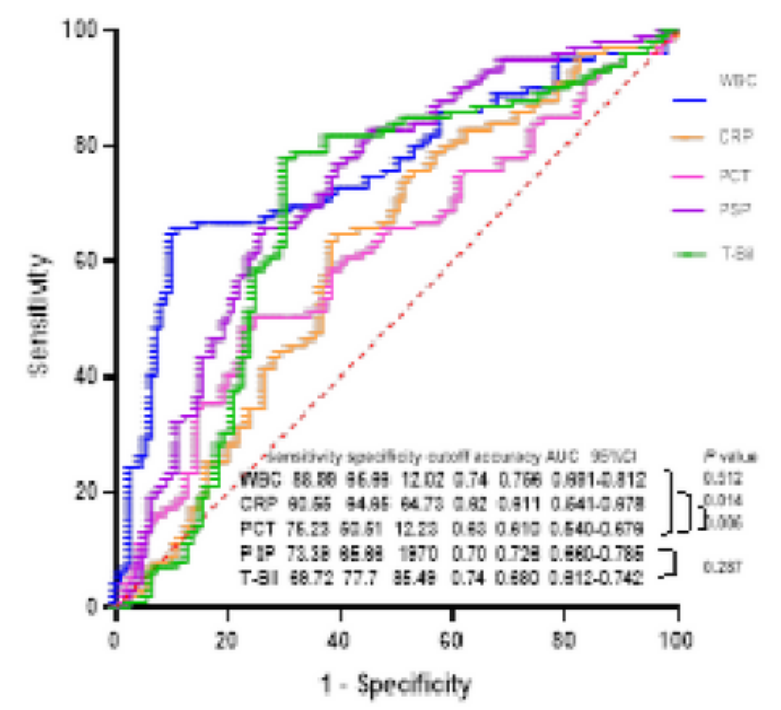

c. ROC curve: ROC of death with acute cholangitis

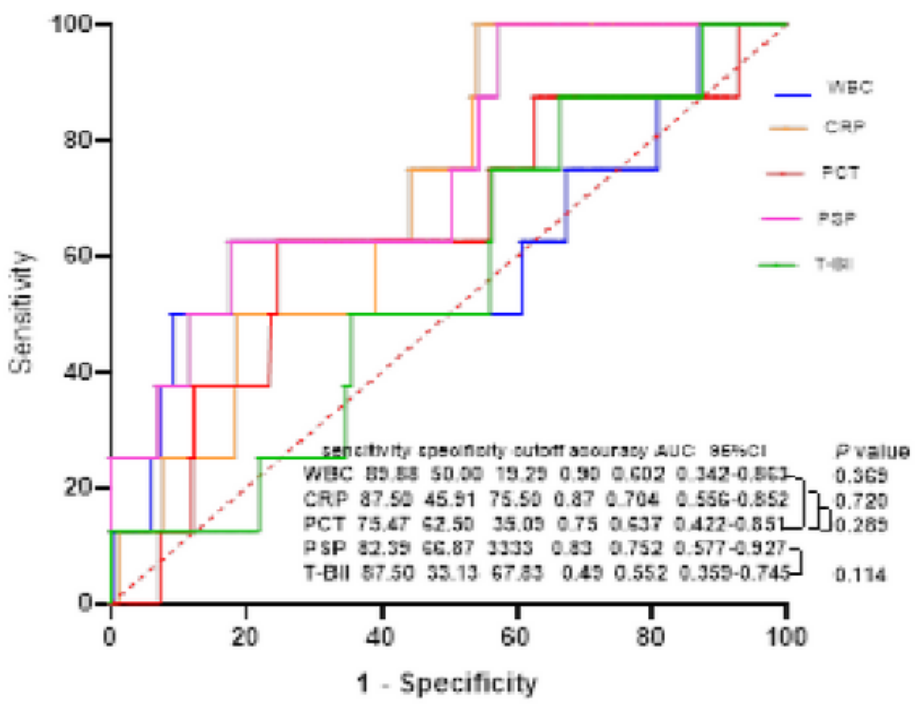

b. ROC curve: ROC of moderate and severe acute cholangitis

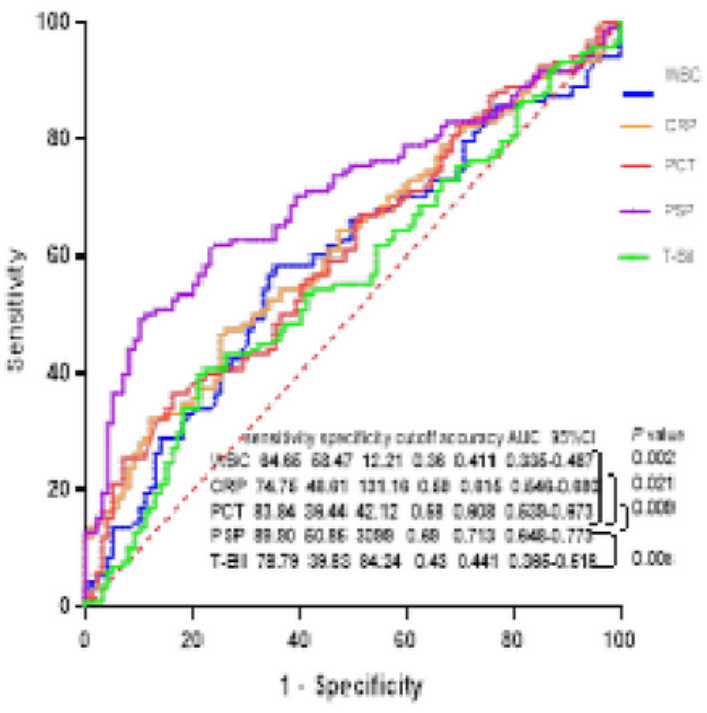

d. ROC curve: ROC of positive blood culture in acute cholangitis

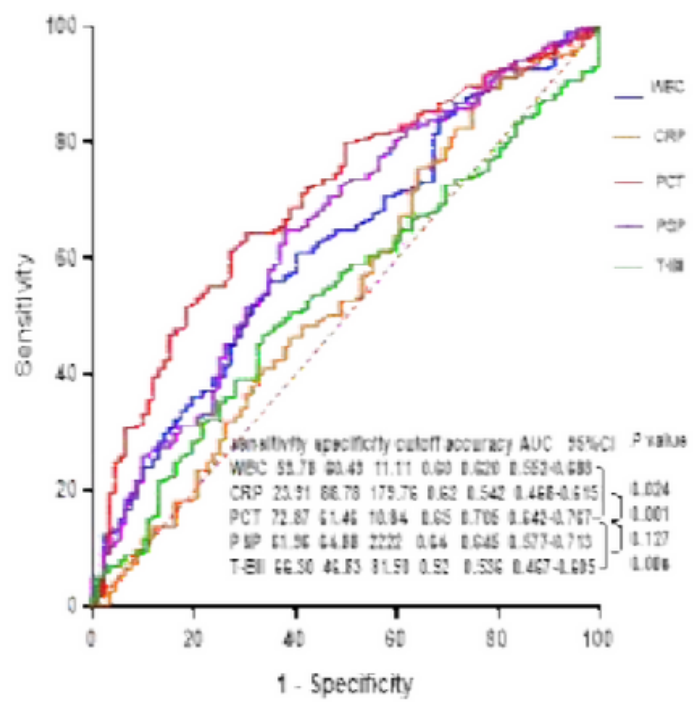

\section{Figure 3}

Receiver operating characteristic curves for white blood cell (WBC), C-reactive protein (CRP), procalcitonin (PCT), presepsin (PSP) and total bilirubin (T-Bil) for classification of severity, prediction of prognosis and positive blood culture in acute cholangitis. The Delong test computed the $P$ value for comparison of the area under the curve (AUC). a. Mild acute cholangitis versus moderate acute cholangitis. b. Moderate acute cholangitis versus severe acute cholangitis. c. The 28-day mortality in acute cholangitis. d. Positive blood culture in acute cholangitis. $P<0.05$ was set to significant level. 

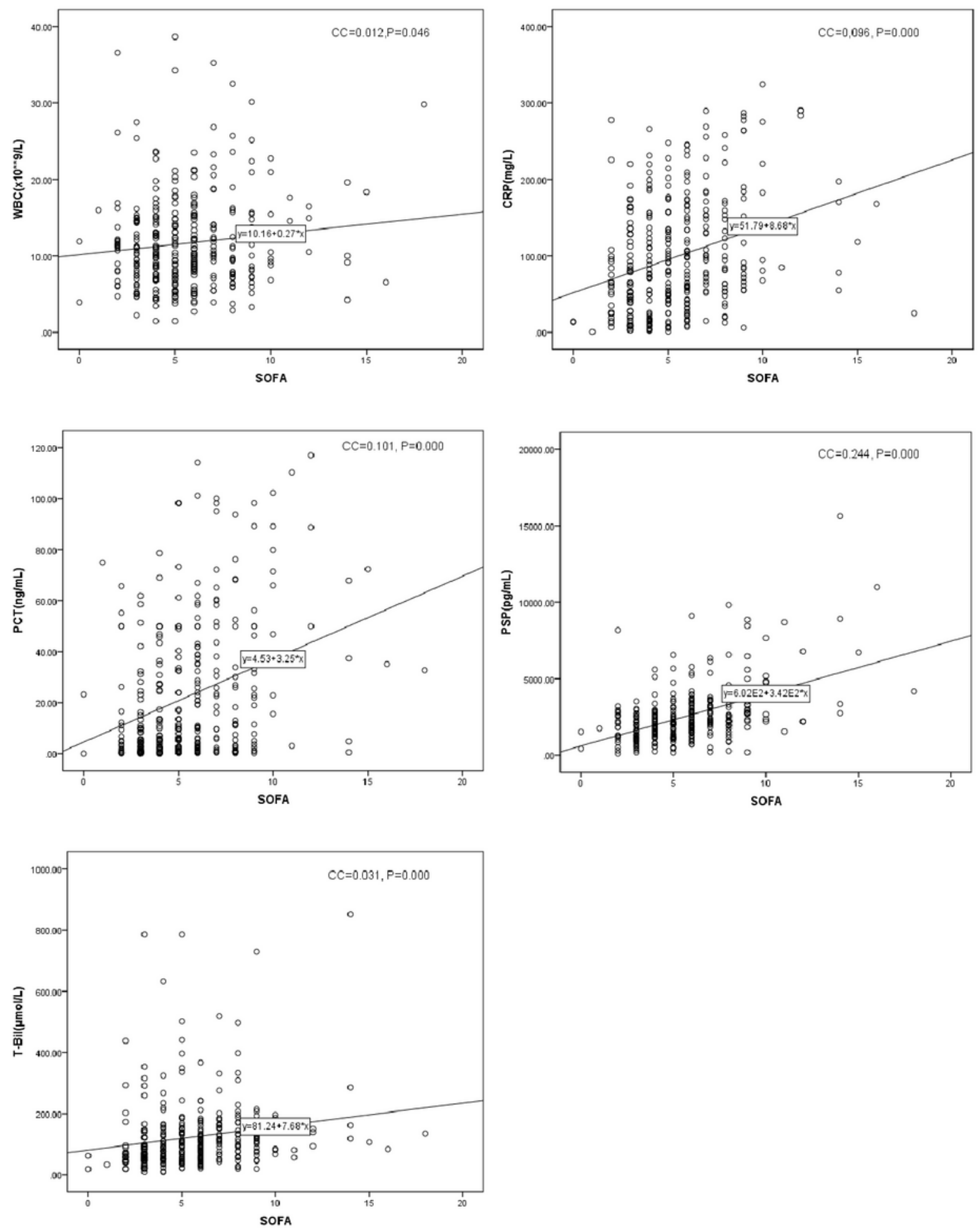

\section{Figure 4}

Correlation between white blood cell (WBC), C-reactive protein (CRP), procalcitonin (PCT), presepsin (PSP), total bilirubin (T-Bil) and Score and sequential organ failure assessment (SOFA). PSP had the best positively correlated with SOFA among them. $\mathrm{P}<0.05$ was set to significant level. 

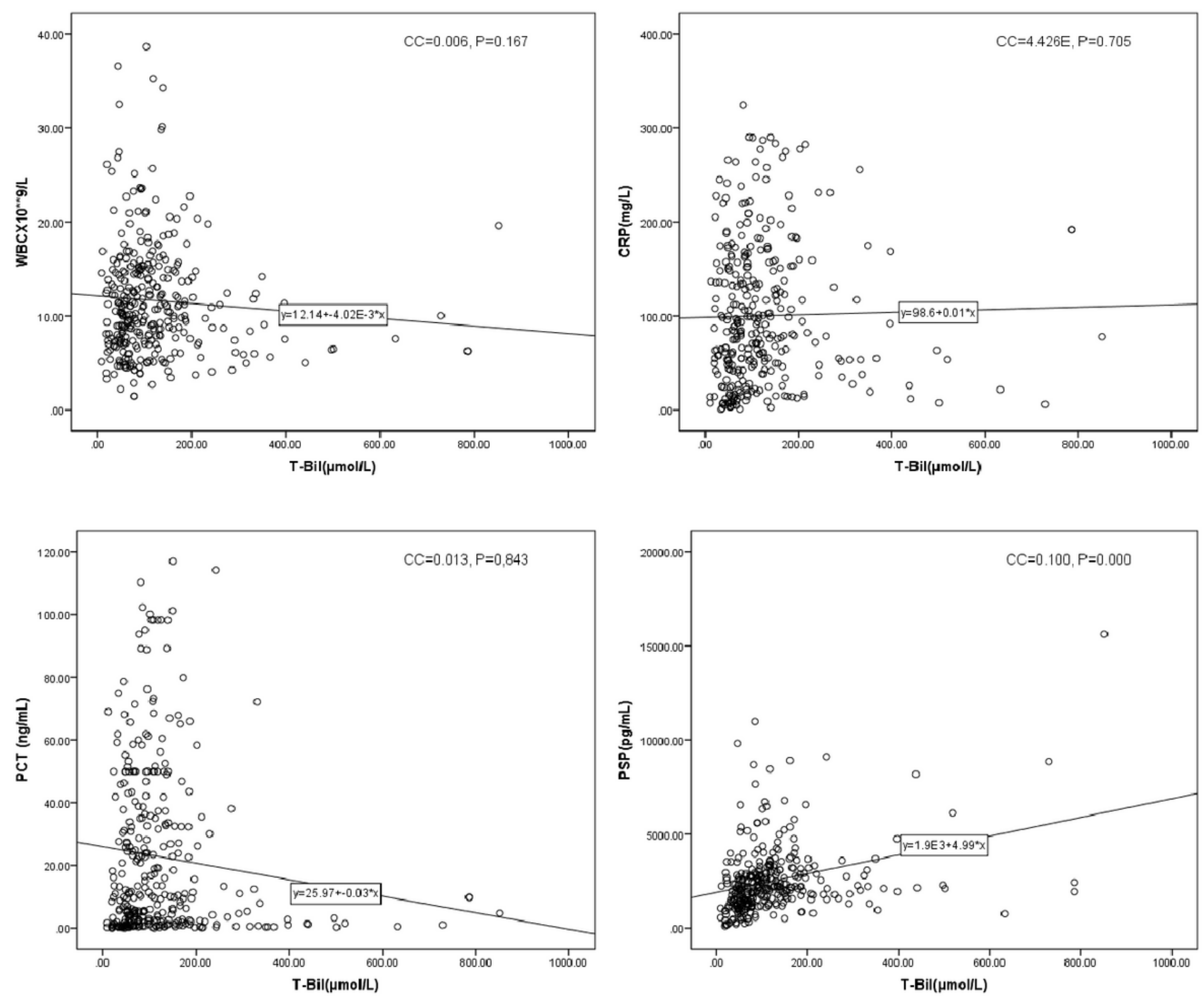

\section{Figure 5}

Correlation between white blood cell (WBC), C-reactive protein (CRP), procalcitonin (PCT), presepsin (PSP) and total bilirubin (T-Bil). Only PSP had positively correlated with T-Bil among them. $\mathrm{P}<0.05$ was set to significant level.

\section{Supplementary Files}

This is a list of supplementary files associated with this preprint. Click to download.

- Table1.pdf

- Table2.pdf 